\title{
Devices for all-optical wavelength conversion and spectral inversion
}

\author{
Antonio Mecozzi \\ Fondazione Ugo Bordoni, via B. Castiglione 59, I-00142 Roma, Italy \\ Phone: +39 (6) 5480--2232 Fax: +39 (6) 5480--4402
}

\begin{abstract}
Recent progress towards efficient frequency converters and spectral inverters based on semiconductor optical amplifiers is reviewed. Criteria for the optimization of the amplifier structure for the particular use are given. Special attention is paid to techniques based on four-wave mixing used for both wavelength conversion and dispersion compensation.
\end{abstract}

\section{Summary}

The last few years have witnessed a tremendous increase in the capacity of point-topoint optical communication systems, both in laboratory experiments and in deployed systems. It is not clear at the moment how much of the bandwidth potentially available from the point-to-point technology will be immediately exploited at the network level. One of the main limitations to the full use of the poin-to-point bandwidth is the speed of the devices used for the processing at the network nodes. Although other options are currently investigated, it is generally acknowledged that a higher capacity will be achieved by the use of devices that process the signal at the optical level, with no need of electronic regeneration. Since the main limit to a high network capacity is the accumulation of noise and signal distorsion induced by the nonlinearity, it is easy to anticipate that the ultimate network capacity will be reached by the use of optical $R^{3}$ regenerators, capable of reshape, retime and reamplification of the signal. Many applications, however, require a linear transfer function. For linear transfer function, we mean that the amplitude of the output signal is proportional to the amplitude of the input signal, and the its phase is either equal or opposite to the phase of the input. Among the applications requiring linearity of the transfer function of the device, we mention the simultaneous processing of many wavelength division 
multiplexed channels or the processing of analog signals. Regeneration of the signal is obviously impossible by linear devices, as reshaping necessarily requires a nonlinear tranfer function.

In this presentation, we will review the recent progress towards the realization of devices for all optical signal processing. Two functionalities will be mainly discussed. One is wavelength conversion, and we will focus on device based on fourwave mixing and cross-phase and cross-gain modulation in semiconductor optical amplifiers. The other is spectral inversion based on four-wave mixing in semiconductor optical amplifiers for dispersion compensation by mid-span spectral inversion. Devices based on four-wave mixing have linear transfer function and are fully transparent to the modulation format, those based on cross-gain and cross-phase modulation have nonlinear transfer function and are not transparent. On the other hand, some of the devices based on cross-phase modulation in interferometric configurations produce a partial reshaping of the signal, although not its retiming.

It is generally acknowledged that semiconductor optical amplifiers are promising candidates as efficient and compact devices for four-wave mixing applications. The converter performance is characterized by the efficiency and by the noise added by the conversion process. We define the efficiency as the ratio between the power of the converted output $P_{2}(L)$ and the power of the input signal $P_{1}(0)$. The efficiency of FWM in semiconductor optical amplifiers has already been studied in details [1,2] In Ref. [2] the simple formula for the efficiency was given

$$
\eta=\frac{P_{2}(L)}{P_{1}(0)}=f \mid R(\Omega) P^{2}\left(\ln \frac{G_{0}}{G}\right)^{2}
$$

where $t=1 /\left[1+P_{1}(0) / P_{0}(0)\right]^{2}, P_{0}(0)$ is the pump power, $R(\Omega)$ is the material response function, which depends on the parameters characterizing the semiconductor nonlinearity and on the frequency detuning between pump and signal $\Omega /(2 \pi)$ and $G_{0}$ and $G$ are the unsaturated and the saturated gain of the amplifier. By this equation, it is possible to show the existence of a pump power corresponding to a maximum of the 
efficiency. Furthermore, it is possible to optimize the device parameters to get high conversion efficiency. In Ref.[3], by using a device $1 \mathrm{~mm}$ long, a conversion efficiency larger than $0 \mathrm{~dB}$ was reported for a conversion interval (the detuning between the input and converted signal) as large as $2 \mathrm{THz}$ for frequency upconversion, $1 \mathrm{THz}$ for frequency downconversion. With a $1.5 \mathrm{~mm}$ and $2.0 \mathrm{~mm}$ devices, the efficiency and the noise characteristic of the device are even better $[4,5]$. It should be noted that the linear gain of semiconductor optical amplifiers does not indefinitely grow with distance as it might be expected, it shows instead a tendency to saturation. This effect is caused by the increasing gain saturation caused by the broadband amplified spontaneous emission. For this reason, amplifiers longer than few hundreds of microns have never been fabricated for linear applications. A semiconductor optical amplifier used for four-wave mixing is however always operating under strong gain saturation from the optical pump, and it is therefore expected that the efficiency grows with the device length, despite the fact that the linear characteristics degrade. Work at Fondazione Ugo Bordoni lead to the prediction that long amplifiers have high performance for nonlinear applications. As an outcome of a collaboration with FUB, the small Swiss manifacturer OptoSpeed has been the first to produce and to make commercially available long amplifiers for this particular use. To date, also Alcatel produces amplifiers longer than $1 \mathrm{~mm}$ specifically designed for nonlinear operation. The world record performance in terms of both efficiency and signal-to noise ratio of a four-wave mixing converter is presently detained by a $2 \mathrm{~mm}$ long multi-quantum-well device fabricated by British Telecom [6].

Another technique for frequency conversion is based on cross-gain and cross-phase modulation. Semiconductor optical amplifiers in interferometric configurations have shown excellent performance for frequency conversion. Work at FUB in collaboration with British Telecom has shown that, surprisingly, the waveguide internal loss is beneficial to increase the extinction ratio of the frequency converted signal $[7,8]$. Recent work at Alcatel has shown that an optimization of the amplifier performance as frequency converter is indeed possible by a careful engineering of the internal loss of the amplifier [9]. 
The most promising application of four-wave mixing in semiconductor optical amplifiers is dispersion compensation, obtained inverting the spectrum of an optical signal midway between the transmitted and the receiver. One of the problem of midspan spectral inversion is the waste of bandwidth caused by the fact that the converted signal is centered at a different frequency than the input. A method to obtain spectral inversion without frequency shift based on the use of two orthogonal pumps in a semiconductor optical amplifier has been recently proposed at FUB $[10,11]$. Independently, K. Inoue at Nippon Telephon and Telegraph came out with essentially the same scheme using four-wave mixing in optical fibers [12].

\section{References}

[1] J. Zhou, N. Park, J. W. Dawson, K. J. Vahala, and M. A. Newkirk, "Efficiency of broadband four-wave mixing wavelength conversion using semiconductor traveling-wave amplifiers," IEEE Photon. Technol. Lett. 6, 50-52 (1994).

[2] A. Mecozzi, "Analytical theory of four-wave mixing in semiconductor amplifiers," Opt. Lett. 19, 892-894 (1994).

[3] A. D'Ottavi, F. Martelli, P. Spano, A. Mecozzi, S. Scotti, R. Dall'Ara, J. Eckner, and G. Guekos, "Very high efficiency four-wave mixing in a single semiconductor traveling-wave amplifier," Appl. Phys. Lett. 68, 2186-2188 (1996).

[4] F. Girardin, J. Eckner, G. Guekos, R. Dall'Ara, A. Mecozzi, A. D'Ottavi, F. Martelli, S. Scotti, and P. Spano, "Low-noise and very high-efficiency four-wave mixing in 1.5-mm-long semiconductor optical amplifiers," IEEE Photon. Technol. Lett. 9, 746-748 (1997).

[5] A. D'Ottavi, F. Girardin, L. Graziani, F. Martelli, P. Spano, A. Mecozzi, Scotti, R. Dall'Ara, J. Eckner, and G. Guekos, "Four wave mixing in semiconductor optical amplifiers: a practical tool for wavelength conversion,” IEEE J. Sel. Top. Quantum Electron. 3, 522-528 (1997). 
[6] D. D Marcenac, D. Nesset, A. E. Kelly, M. Briely, A. D. Ellis, D. G. Moodie, and C. W. Ford, "40 Gbit/s transmission over $406 \mathrm{~km}$ of NDSF using midspan spectral inversion in a $2 \mathrm{~mm}$ long semiconductor optical amplifier," Electron. Lett. 33, 879-881 (1997).

[7] A. Mecozzi, "Small signal theory of wavelength converters based on cross-gain modulation in semiconductor optical amplifiers," IEEE Photon. Technol. Lett. 8, 1471-1473 (1996). A. Mecozzi and J. Mørk, "Saturation effects in nondegenerate four-wave mixing between short optical pulses in semiconductor optical amplifiers," J. Sel. Topics Quantum Electron. 3, 1190-1207 (1997).

[8] D. Marcenac and A. Mecozzi, "Switches and frequency converters based on cross-gain modulation in semiconductor optical amplifiers," IEEE Photon. Technol. Lett. 9, 749-751 (1997).

[9] T. Ducellier, "Integrated GAG-SOA (Gain-Attenuation-Gain) for ultra-high speed wavelength conversion," SOA based components for optical networks workshop, Praha, October 27-28, 1997.

[10] A. Mecozzi, Italian patent No. RM 96 A 000786, November 18, 1996.

[11] A. Mecozzi, G. Contestabile, F. Martelli, L. Graziani, A. D'Ottavi, P. Spano, R. Dall'Ara, and J. Eckner, F. Girardin, and G. Guekos, "Optical spectral inversion without frequency shift by four-wave mixing using two pumps with orthogonal polarizations," IEEE Photon. Technol. Lett. 10, 335-337 (1998).

[12] K. Inoue, "Spectral inversion with no wavelength shift based on four-wave mixing with orthogonal pump beams," Opt. Lett. 22, 1772-1774, (1997). 